\title{
Multiple Higgs and vector boson production beyond the Standard Model
}

\author{
A. Belyaev, ${ }^{a, b}$ A.C.A. Oliveira, ${ }^{c, d}$ R. Rosenfeld ${ }^{c, d}$ and M.C. Thomas ${ }^{a}$ \\ ${ }^{a}$ School of Physics $\&$ Astronomy, University of Southampton, \\ Highfield, Southampton SO17 1BJ, U.K. \\ ${ }^{b}$ Particle Physics Department, Rutherford Appleton Laboratory, \\ Chilton, Didcot, Oxon OX11 0QX, U.K. \\ ${ }^{c}$ Instituto de Física Teórica - Universidade Estadual Paulista and \\ ICTP South American Institute for Fundamental Research, \\ Rua Dr. Bento T. Ferraz 271, 01140-070, São Paulo, Brazil \\ ${ }^{d}$ Theory Division, Physics Department, CERN, \\ CH-1211, Geneva 23, Switzerland \\ E-mail: a.belyaev@soton.ac.uk, alexandra.oliveira@cern.ch, \\ rosenfel@ift.unesp.br, mct1g11@soton.ac.uk
}

ABSTRACT: If the electroweak symmetry breaking is originated from a strongly coupled sector, as for instance in composite Higgs models, the Higgs boson couplings can deviate from their Standard Model values. In such cases, at sufficiently high energies there could occur an onset of multiple Higgs boson and longitudinally polarised electroweak gauge boson $\left(V_{L}\right)$ production. We study the sensitivity to anomalous Higgs couplings in inelastic processes with 3 and 4 particles (either Higgs bosons or $V_{L}$ 's) in the final state. We show that, due to the more severe cancellations in the corresponding amplitudes as compared to the usual $2 \rightarrow 2$ processes, large enhancements with respect to the Standard Model can arise even for small modifications of the Higgs couplings. In particular, we find that triple Higgs production provides the best multiparticle channel to look for these deviations. We briefly explore the consequences of multiparticle production at the LHC.

Keywords: Higgs Physics, Beyond Standard Model, Technicolor and Composite Models, Standard Model 


\section{Contents}

1 Introduction 1

2 Multiparticle cross sections and unitarity 2

3 Anomalous Higgs couplings and partial unitarization 3

4 Sensitivity of $2 \rightarrow 3,4$ cross section to anomalous couplings 5

5 Cross sections in the SM with anomalous Higgs couplings 9

6 Impact of multiparticle production at the LHC and future colliders 10

$\begin{array}{llr}7 \text { Conclusion } & 11\end{array}$

\section{Introduction}

The search for the mechanism responsible for electroweak symmetry breaking (EWSB) has been a long-lasting endeavour that may finally be resolved with the new data from the LHC. However, even with the recent evidence for a light scalar state, the jury is still out on whether EWSB is caused by new strong interactions or not. In order to have a definitive answer further experimental probes are necessary.

In particular, a hallmark of strong interactions in the EWSB sector is multiple particle production [1]. ${ }^{1}$ In a strongly coupled EWSB sector one would expect copious production of longitudinal gauge bosons granted enough energy is available to produce them. This would be similar to the production of events with a large pion multiplicity in QCD at high energies. In fact, multi-W production was studied in a simplified scaled-up version of QCD almost 20 years ago [3]. In this article we study the inelastic production of longitudinally polarised $\mathrm{W}$ and $\mathrm{Z}$ bosons (denoted collectively by $V_{L}$ ) and Higgs bosons in the context of an effective Lagrangian. We estimate the energy scale at which these processes become relevant, which signals the onset of new physics, as recently discussed in [4]. In particular, we will be interested in the sensitivity to non-SM Higgs couplings in the growth of the cross section for these processes. Our results have as a particular case the study of unitarity violation in multi- $V_{L}$ production in the Higgsless model [5]. We show that models with partial unitarization, such as the composite Higgs model, can lead to a large enhancement of multiparticle cross section due to the absence of cancellation mechanisms in the corresponding scattering amplitudes. This effect becomes more acute as the final state multiplicity increases, provided that enough energy is available.

\footnotetext{
${ }^{1}$ One should note, however, that multiple particle production with large cross section could also be obtained in weakly coupled theories at tree level simply due to the large number of diagrams, but this is expected only for very large multiplicities, of order $\mathcal{O}\left(1 / \alpha_{E W}\right)[2]$.
} 


\section{Multiparticle cross sections and unitarity}

The perturbative unitarity bound in the inelastic $2 \rightarrow n$ process assuming $s$-wave dominance for a given center-of-mass energy $\sqrt{s}$ is $[5,6]$ :

$$
\sigma(2 \rightarrow n)<\frac{4 \pi}{s}
$$

This unitarity bound sets stringent constraints on the scattering amplitudes. Since the relativistic $n$-body phase space is proportional to $s^{n-2}$ the unitarity bound requires that the amplitude grows with energy no faster than

$$
A(2 \rightarrow n) \sim s^{1-n / 2}
$$

Suppose, for instance, that the EWSB sector is described by a simple nonlinear sigma model $(\mathrm{NL} \sigma \mathrm{M})$, neglecting transverse gauge bosons for the moment, and assuming possible resonance states to be very heavy:

$$
\mathcal{L}_{N L \sigma M}=\frac{v^{2}}{4} \operatorname{Tr}\left[\partial_{\mu} U \partial^{\mu} U^{\dagger}\right]
$$

where $v=246 \mathrm{GeV}$ is the usual scale of electroweak symmetry breaking and

$$
U=e^{\frac{i \vec{r} \cdot \vec{\pi}}{v}} .
$$

The isospin triplet "pion" fields $\pi^{i}(i=1,2,3)$ will be identified with the longitudinally polarised gauge bosons through the equivalence theorem [1]. By power-counting, the scattering amplitude in this model grows with energy as

$$
A_{N L \sigma M}(2 \rightarrow n) \sim \frac{s}{v^{n}}
$$

and hence naively

$$
\sigma(2 \rightarrow n) \sim \frac{1}{s}\left(\frac{s}{v^{n}}\right)^{2} s^{n-2} .
$$

Therefore, the growth of the cross section towards the unitarity bound in this model is faster for larger number of particles due to the kinematical factors in the phase space, assuming of course that enough energy is available.

Conversely, there must be stronger cancellations in the scattering amplitudes due to new physics as the number of final state particles is increased. For instance, unitarity requires that $A(2 \rightarrow 2) \sim$ constant and $A(2 \rightarrow 4) \sim 1 / s$, whereas they both grow as $\sim s$ in the NL $\sigma \mathrm{M}$. Therefore, in the absence of a perfect cancellation, it is plausible that the growth of the cross section may have a large impact in multi- $V_{L}$ production. It is the purpose of this work to examine this impact.

Given the fully relativistic $n$-body phase space given by: ${ }^{2}$

$$
R_{n}(s)=\int \prod_{i=1}^{n} \frac{d^{3} p_{i}}{(2 \pi)^{3}\left(2 E_{i}\right)^{3}}(2 \pi)^{4} \delta^{4}\left(\sqrt{s}-\sum_{i=1}^{n} p_{i}\right)=\frac{(2 \pi)^{4-3 n}(\pi / 2)^{n-1}}{(n-1) !(n-2) !} s^{n-2}
$$

\footnotetext{
${ }^{2}$ See e.g. [7].
} 
one can easily estimate the energy scale $\Lambda_{n}$ at which perturbative unitarity is violated in $2 \rightarrow n$ processes in the NL $\sigma \mathrm{M}$ :

$$
\Lambda_{n}=\left[\frac{2(n-1) !(n-2) !}{(2 \pi)^{3-3 n}(\pi / 2)^{n-1}}\right]^{\frac{1}{2 n}} v .
$$

For example, unitarity is violated in $2 \rightarrow 4$ processes at an energy which is almost 2.5 higher than that for the usual $2 \rightarrow 2$ processes. This estimate is in reasonable agreement with [5]. One should notice that we are not including in this rough estimate the growth due to the combinatorial factors and a proper phase space integration. These will be included below in a fully automated calculation.

\section{Anomalous Higgs couplings and partial unitarization}

In order to recover unitarity there must an UV completion of the model describing the interaction of the lightest degrees of freedom. The simplest possibility is the addition of a scalar field, which is identified with the Higgs scalar. However, it is possible that the Higgs scalar is a composite particle with couplings that may differ from the SM ones. In this case, the theory is not UV-complete and unitarity is only partially restored. For such a theory one can use an effective Lagrangian to parameterise its couplings to longitudinally polarised gauge bosons and self-couplings (couplings to fermions are not relevant to the results presented here) [8]:

$$
\begin{aligned}
\mathcal{L}_{\text {eff }}= & \frac{v^{2}}{4}\left(1+2 a \frac{h}{v}+b \frac{h^{2}}{v^{2}}+b_{3} \frac{h^{3}}{v^{3}}+\cdots\right) \operatorname{Tr}\left[\partial_{\mu} U \partial^{\mu} U^{\dagger}\right] \\
& +\frac{1}{2}\left(\partial_{\mu} h\right)^{2}-\frac{1}{2} m_{h}^{2} h^{2}-d_{3} \lambda v h^{3}-d_{4} \frac{\lambda}{4} h^{4}+\cdots
\end{aligned}
$$

This parameterisation is common to a large class of models, such as composite Higgs models, and has been used to study anomalous Higgs couplings in $V_{L} V_{L} \rightarrow V_{L} V_{L}$, hh processes at the LHC $[8,10,11]$. Unitarity is recovered for the SM values $a=b=d_{3}=d_{4}=1$ and $b_{3}=0$. For different values of these parameters the usual cancellation provided by the scalar field is incomplete. As an example, the Minimal Composite Higgs Model (MCHM4) predicts that the couplings of the "pions" with the Higgs boson follows from an expansion around the vacuum $h(x)=0$ of the effective Lagrangian [12]

$$
\frac{f^{2}}{4} \sin ^{2}\left(\theta+\frac{h(x)}{f}\right) \operatorname{Tr}\left[\partial_{\mu} U \partial^{\mu} U^{\dagger}\right]
$$

with the identification $v=f \sin \theta$, which comes from the mass term for the gauge fields. This Lagrangian has a discrete symmetry under the parity transformation $h \rightarrow-h$ and $\pi \rightarrow-\pi$, although this is not obvious in this representation [12]. Therefore, in the MCHM4

$$
a=\sqrt{1-\xi} ; \quad b=1-2 \xi ; \quad b_{3}=-\frac{4}{3} \xi \sqrt{1-\xi} ; \quad \cdots
$$


In order to study the $2 \rightarrow 4$ scattering, one must expand each field $U$ to order $\mathcal{O}\left(\pi^{6}\right)$ :

$$
\begin{aligned}
\frac{v^{2}}{4} \operatorname{Tr}\left[\partial_{\mu} U \partial^{\mu} U^{\dagger}\right]= & \frac{1}{2}\left(\partial^{\mu} \vec{\pi} \cdot \partial_{\mu} \vec{\pi}\right)+\left[1-\frac{2}{15 v^{2}} \vec{\pi} \cdot \vec{\pi}\right] \\
& \times \frac{1}{6 v^{2}}\left[\left(\vec{\pi} \cdot \partial_{\mu} \vec{\pi}\right)^{2}-(\vec{\pi} \cdot \vec{\pi})\left(\partial^{\mu} \vec{\pi} \cdot \partial_{\mu} \vec{\pi}\right)\right]+\mathcal{O}\left(\vec{\pi}^{8}\right)
\end{aligned}
$$

The number of diagrams increases considerably with the number of final state particles, making it impractical to perform an analytical computation. Hence we have implemented the Lagrangian given in eq. (3.1) in FormCalc [13] and MadGraph [14] using FeynRules [15] (with UFO output [16] for the higher dimensional operators). We have also implemented this model in CalcHEP [17] using LanHEP package [18] with the help of auxiliary fields. This Lagrangian is equivalent to the usual linear sigma model by a field redefinition.

In the familiar case of $2 \rightarrow 2$ amplitudes the only kinematical dependence is on the Mandelstam variables $s$ and $t$. For instance, denoting the goldstone bosons by their electric charge, the $00 \rightarrow+-$ amplitude, arising from only 4 diagrams, is given by:

$$
\mathcal{M}_{00 ;+-}=\frac{s\left[\left(1-a^{2}\right) s-m_{h}^{2}\right]}{v^{2}\left(s-m_{h}^{2}\right)} \underset{s \gg m_{h}^{2}}{\longrightarrow}\left(1-a^{2}\right) \frac{s}{v^{2}}
$$

Hence one can easily see that there is a violation of unitarity even with the presence of the Higgs boson if its coupling is not SM-like, i.e., $a \neq 1$. However, in the SM one obtains a constant amplitude at high energies, as expected.

The $2 \rightarrow 4$ amplitudes are much more complicated, containing of the order of 100 diagrams and depending on several combinations of the scalar products of the different 4-momenta involved. However, some of their properties can be demonstrated with the following simple example for a given point in phase space, where all the particles lie in the same plane with an angle of $\pi / 3$ between the nearest neighbours (we will keep the "pions" massless at the amplitude level since their masses are not relevant for issues of unitarity), in which case we obtain

$$
\begin{aligned}
\mathcal{M}_{00 ; 00+-} \propto \frac{1}{v^{4}}[ & 72 s\left(13 a^{4}-a^{2}(7 b+5)-1\right)+ \\
& +3 m_{h}^{2}\left(1580 a^{4}-378 a^{3} d_{3}-3 a^{2}(245 b+131)-74\right)+ \\
& +\frac{m_{h}^{4}}{s}\left(9774 a^{4}-3087 a^{3} d_{3}-a^{2}(4494 b+1289)+52\right)+ \\
& +\cdots]
\end{aligned}
$$

It grows with $s$, as expected. However, in the $\mathrm{SM}\left(a=b=d_{3}=1\right)$ one obtains in the limit $s \gg m_{h}^{2}$ :

$$
\mathcal{M}_{00 ; 00+-} \propto \frac{1}{s} \frac{m_{h}^{4}}{v^{4}}
$$

and we explicitly see the strong cancellation where the first two terms in the amplitude vanish and the behaviour change from $s / v^{4}$ to $m_{h}^{4} /\left(s v^{4}\right)$, as anticipated. The triple Higgs anomalous coupling parameterised by $d_{3}$ does not enter in the dominant contribution. In 
the following we will take $d_{3}=1$. There is no contribution from the couplings $d_{4}$ and $b_{3}$ for the above processes. This result for the amplitude depends on the phase space configuration. The polynomials will be different but the features described above also happens at other phase space configurations and other channels.

For $2 \rightarrow 3$ processes a similar analysis can be performed. For instance, again for a given configuration in phase space where all particles lie in the same plane we find

$$
\begin{aligned}
\mathcal{M}_{00 ; h h h} \propto \frac{1}{4 v^{3}}[ & \left.s\left(-4 a^{3}+4 a b-3 b_{3}\right)\right) \\
& -m_{h}^{2}\left(-8 a^{3}+8 a b+3 b_{3}\right)+ \\
& \left.+\frac{4 m_{h}^{4}}{s}\left(a^{3}+a b-6 b_{3}-3 a^{2} d_{3}\right)+\cdots\right]
\end{aligned}
$$

for the angles between the nearest Higgs bosons fixed at $2 \pi / 3$ and

$$
\begin{aligned}
\mathcal{M}_{00 ;+-h} \propto \frac{a}{192 v^{3}}[ & s\left(-1+2 a^{2}-b\right)+ \\
& +\frac{m_{h}^{2}}{4}\left(-164+386 a^{2}-213 b-9 a d_{3}\right) \\
& \left.-\frac{3 m_{h}^{4}}{2 s}\left(-262+291 a^{2}-93 b+81 a d_{3}\right)+\cdots\right]
\end{aligned}
$$

for the fixed configuration of two collinear pions, back-to-back with the Higgs boson and at right angles with the incident particles.

We again find that for the SM the first two terms in these amplitudes vanish, as it should. Notice also that the $\mathcal{M}_{00 ; h h h}$ amplitude is sensitive to $b_{3}$, being the lowest multiplicity process in which this happens. In addition, as can be anticipated from the parity of the MCHM4 class of theories, under which $\pi \rightarrow-\pi$ and $h \rightarrow-h$ [12], the polynomial with largest growth in the $2 \rightarrow 3$ processes also vanish for the values of $a, b$ and $b_{3}$ that obey the MCHM4 relations as in eq. (3.3).

In summary, for $a \neq 1$ and $b \neq 1$ the squared amplitude grows as $s^{2}$ instead of decreasing as $1 / s^{2}$. Therefore there is a large sensitivity of $2 \rightarrow 3$ and $2 \rightarrow 4$ processes to non-SM Higgs couplings compared to $2 \rightarrow 2$ processes, whereas the SM amplitude goes to a constant for large $s$. For the $2 \rightarrow 3$ processes, there is a also a suppression if the values for the parameters predicted by the MCHM4 are used due to the symmetry of the coset. In order to quantify this sensitivity we study directly the cross section for these processes in the next section.

\section{Sensitivity of $2 \rightarrow 3,4$ cross section to anomalous couplings}

In this section we analyse the cross section for the $2 \rightarrow 3,4$ processes at the parton level for couplings using the Lagrangian eq. (3.1) implemented in CalcHEP. We studied several different channels but will report only on the most representative ones.

In order to show examples of the enhancements that result from the anomalous Higgs couplings, we compute cross sections with a Higgs mass $m_{h}=125 \mathrm{GeV}$. We implement a cut in the invariant mass of the final state pions $m_{+-}>200 \mathrm{GeV}$, such that the Higgs is never 
on-mass-shell when coupled to two pions, as is the case in the SM (the Higgs does not decay to a on-shell pair of gauge bosons). We show in figure 1 the ratio of the cross section as a function of $a$ (keeping the other parameters fixed in one case and, in the case of MCHM4, changing them according to eq. (3.3)) to the SM cross section at a fixed center-of-mass energy of $\sqrt{s}=1 \mathrm{TeV}$. Several channels are shown, such as $(00,+-) \rightarrow+-,+-h, h h h,+-$ +- . The notation $(00,+-)$ indicates that both 00 and +- initial states have been taken into account in the cross section. The left plot shows results for different values of $a$ but keeping the other parameters fixed at their SM values whereas in the right plot we show results when the other parameters are changed according to the MCHM4 where, in addition to the relations given in eq. (3.3), one assumes $d_{3}=\sqrt{1-\xi}$. Of course not all the values of the anomalous couplings are allowed: this plot is only meant for illustrative purposes.

Large enhancements of the order of $10^{3}-10^{5}$ with respect to the SM value are easily obtained even for small deviations (as small as 10\%) of the couplings from their SM values. The largest case occurs for triple Higgs production. The behaviour of the curves of the left plot are easily understandable: the 4, 3 and 2 dips in the cross section versus $a$ for $2 \rightarrow 4$, $2 \rightarrow 3$ and $2 \rightarrow 2$ are due to the $4^{\text {th }}, 3^{r d}$ and $2^{\text {nd }}$ order polynomials in $a$ in the amplitudes. One can see that the enhancements in $2 \rightarrow 2$ processes are modest compared to processes with higher multiplicities, at least at $1 \mathrm{TeV}$. For the MCHM4 case there is a suppression in the $2 \rightarrow 3$ process, as expected from the parity symmetry of the coset. Since the MCHM4 always predict smaller deviations, in what follows we will consider the more optimistic case where the parameter $a$ can be the only one different from the SM values.

Next we study the growth with center-of-mass energy of the cross section for different multiplicities for a few values of the anomalous coupling, namely $a=0.9,0.95$ and 1 (SM), keeping the other couplings at their SM values. We believe that these values of the anomalous couplings can be representative of the behaviour of the cross sections, that is, we expect the same order-of-magnitude enhancements if the other couplings are also anomalous (but without obeying the MCHM4 relations). In figure 2 we present a comparison of the cross section as a function of energy among representative processes with 2, 3 and 4 particles in the final state, for different values of the anomalous coupling. We also show the unitarity limit eq. (2.1). A few comments are in order. The SM cross section quickly stabilises at a small value, which depends on the specific process (of the order of $10^{-3} \mathrm{pb}, 10^{-2} \mathrm{pb}$ and $10^{-1} \mathrm{pb}$ for $00 \rightarrow h h h,(00,+-) \rightarrow+-h$ and $(00,+-) \rightarrow+-$, respectively). It is not surprising that in the non-SM case the cross sections grow very fast with energy, reaching up to order of $100 \mathrm{pb}$ and violating unitarity at center-of mass energies of the order of a few $\mathrm{TeV}$. It is also anticipated that larger multiplicity processes, in the absence of a complete cancellation mechanism, grow faster due to phase space. However, what is somewhat unexpected is the energy scale at which multiparticle cross sections become comparable to $2 \rightarrow 2$ processes. In the examples shown the $2 \rightarrow 3$ start to overcome $2 \rightarrow 2$ at energies of $\mathcal{O}(1 \mathrm{TeV})$. This may be signalling the onset of nonperturbative behaviour well before the unitarity bound is reached. It is not clear whether new physics must come in at these scales, as for instance the appearance of new resonances. In this work we assume that this is not the case. We also checked that the $2 \rightarrow 4$ process grows very rapidly for $a \neq 1$, but since it starts out very suppressed it surpasses the $2 \rightarrow 2$ only at very high energies, of the order of $\mathcal{O}(5 \mathrm{TeV})$. 

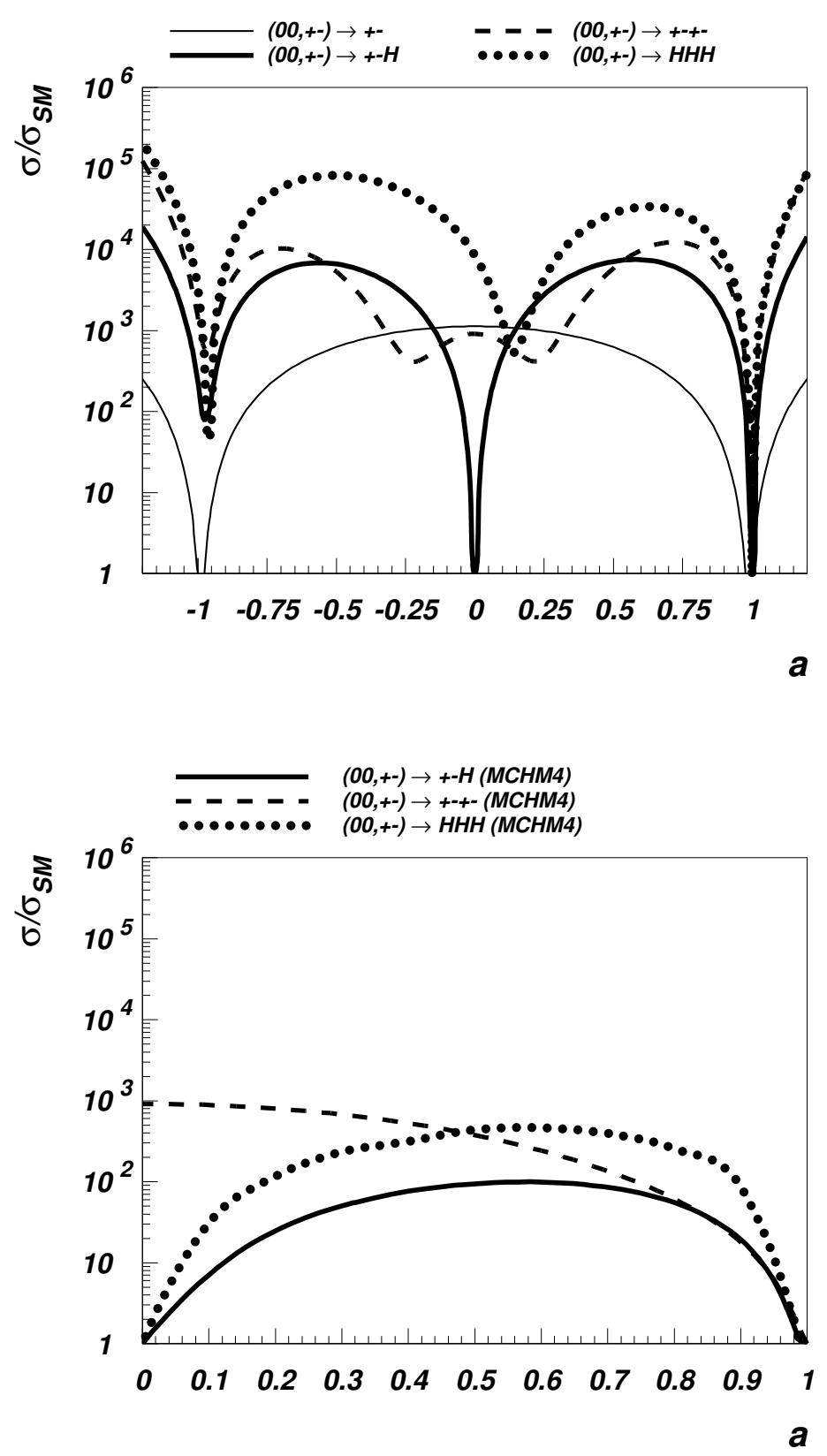

Figure 1. Ratio of cross sections as a function of a parameter to the SM ones for two cases: the general effective lagrangian with all parameters but $a$ fixed at their SM values (left plot) and for MCHM4 model (right plot) at a fixed energy of $\sqrt{s}=1 \mathrm{TeV}$. The different channels are: $(00,+-) \rightarrow+-+-($ dashed line), $(00,+-) \rightarrow+-h$ (thick solid line), $(00,+-) \rightarrow$ hhh (dotted line), and $(00,+-) \rightarrow+-$ (thin solid line) for comparison. The notation $(00,+-)$ indicates that both 00 and +- initial states were taken into account. 


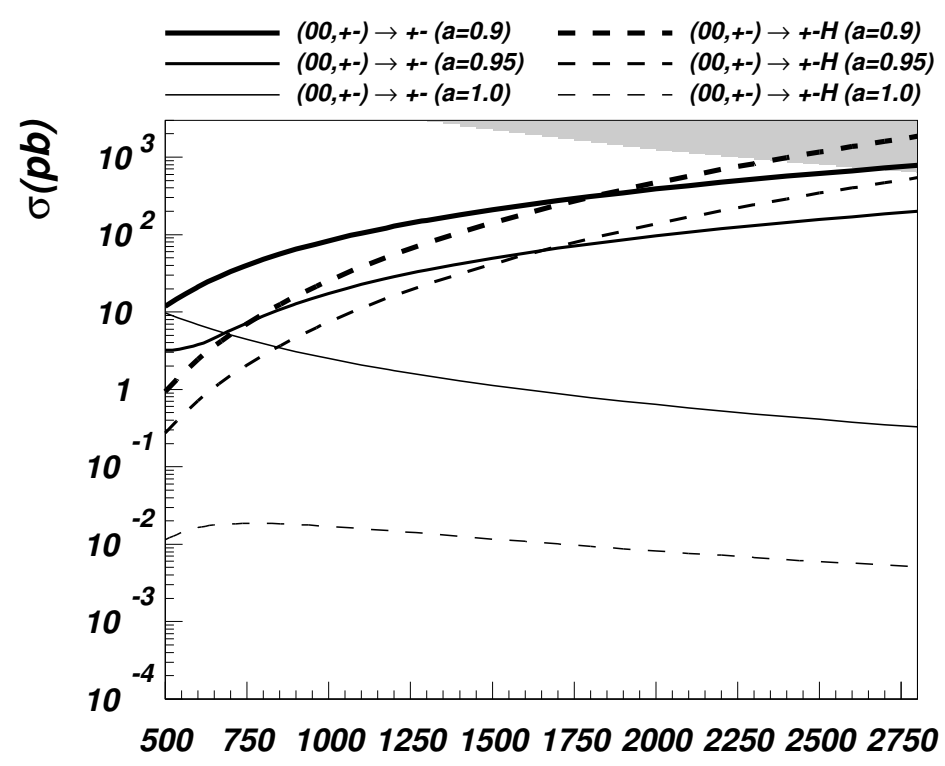

$\sqrt{s}(\mathrm{GeV})$

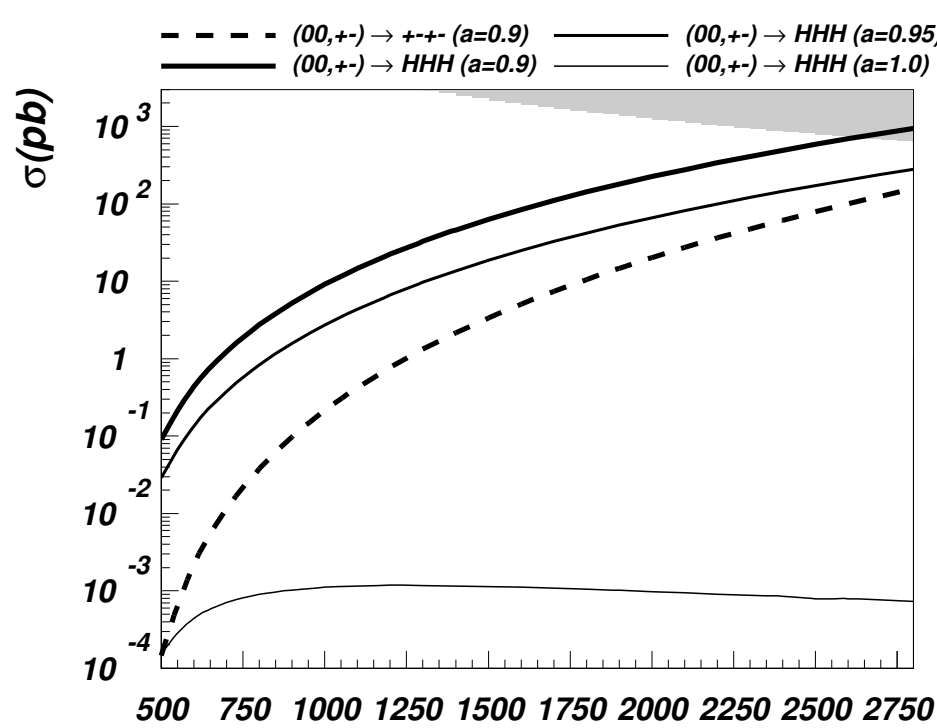

$\sqrt{s}(\mathrm{GeV})$

Figure 2. Comparison among cross sections as a function of the center-of-mass energy for processes with 2,3 and 4 particles in the final state. In the top plot, the solid lines are for $(00,+-) \rightarrow+-$ for $a=0.9$ (thick), $a=0.95$ (medium thick) and $a=1$ (thin). Dashed lines are for $(00,+-) \rightarrow+-h$, with the same pattern for the thickness of the lines. In the bottom plot, the same pattern of lines show the results $(00,+-) \rightarrow h h h$ and the process $00 \rightarrow+-+-$ is shown as a dashed line for $a=0.9$. In these plots only $a$ deviates from the SM value. The unitarity bound is shown as a shaded area in the top right corner. 


\begin{tabular}{|l|l|l|}
\hline channel & $a=b=1(\mathrm{SM})$ & $a=0.9 ; b=1$ \\
\hline $00 \rightarrow+-$ & $0.53(0.13)$ & $66.4(295)$ \\
\hline$Z Z \rightarrow W^{+} W^{-}$ & $629(610)$ & $646(655)$ \\
\hline $00 \rightarrow+-h$ & $4.6 \times 10^{-3}\left(2.0 \times 10^{-3}\right)$ & $18.7(350)$ \\
\hline$Z Z \rightarrow W^{+} W^{-} h$ & $5.49(10.9)$ & $6.17(46.2)$ \\
\hline $00 \rightarrow h h$ & $0.64(0.18)$ & $43.0(158)$ \\
\hline$Z Z \rightarrow h h$ & $7.18(7.61)$ & $4.31(15.7)$ \\
\hline $00 \rightarrow h h h$ & $5.6 \times 10^{-4}\left(4.9 \times 10^{-4}\right)$ & $4.5(112)$ \\
\hline$Z Z \rightarrow h h h$ & $1.7 \times 10^{-2}\left(4.7 \times 10^{-2}\right)$ & $0.61(13.6)$ \\
\hline
\end{tabular}

Table 1. Comparison of $2 \rightarrow 2$ and $2 \rightarrow 3$ cross sections (in picobarns) at $\sqrt{s}=1 \mathrm{TeV}(\sqrt{s}=2 \mathrm{TeV}$ in parenthesis).

\section{Cross sections in the SM with anomalous Higgs couplings}

So far we have only analysed the scattering of the longitudinally polarised gauge bosons. Since it is difficult to separate out the contributions from these polarisations in an experimental setting, it is important to understand how the large enhancements found will affect the corresponding unpolarised cross section. In order to do so, we use the Lagrangian eq. (3.1) promoting the partial derivatives to full covariant derivatives and adopt the unitary gauge $(U=\mathbf{1})$. As an illustration, we fix the partonic center-of-mass energy at $2 \mathrm{TeV}$, which is still below the unitarity bound since there is a partial unitarization due to the presence of a Higgs boson, and compare the cross sections for the longitudinally polarised gauge bosons with the full SM in table 1 . We still keep the notation $0,+,-$ to indicate longitudinally polarised gauge bosons and $Z, W^{ \pm}$to denote the unpolarised gauge bosons.

Notice that the processes with longitudinal polarisations are subdominant in the SM. However, as we discussed above, they are greatly enhanced with small deviations of the couplings and actually dominate the cross sections. The results are consistent with the fact that $\sigma_{\text {all }} \approx \sigma_{L L} / 9$ when the contribution from longitudinal polarisations is dominant. The enhancements are larger when the final state multiplicity is larger, as expected. For instance, $\sigma(00 \rightarrow+-h)>\sigma(00 \rightarrow+-)$ for $a=0.9$ and $b=1$. When all polarisations are included, the contribution from transverse polarisations can mask the increase in the cross section for the longitudinally polarised gauge bosons. This can be seen in the case of $Z Z \rightarrow W^{+} W^{-}$, where the total increase in the cross section is less than $10 \%$. On the other hand, in cases where the contributions from the transverse polarisations are not large, as in the case of $Z Z \rightarrow h h h$, enhancements of $\mathcal{O}\left(10^{3}\right)$ can be obtained. Therefore, multiple Higgs production offers the best channels to study anomalous couplings. For the unpolarised case, the cross section for $Z Z \rightarrow W^{+} W^{-}$is still at least one order of magnitude larger than the typical $2 \rightarrow 3$ processes but $Z Z \rightarrow h h$ is of the same order as the $Z Z \rightarrow h h h$ cross section. In the next section we discuss the impact of these results for the LHC and future colliders. 


\begin{tabular}{|c|c|c|c|c|}
\hline \multirow{4}{*}{ Process } & \multicolumn{2}{|c|}{$14 \mathrm{TeV}$} & \multicolumn{2}{|c|}{$33 \mathrm{TeV}$} \\
\hline & \multicolumn{2}{|c|}{ with (without) VBF cuts } & \multicolumn{2}{|c|}{ with (without) VBF cuts } \\
\hline & $\mathrm{a}=1.0$ & $a=0.9$ & $a=1.0$ & $a=0.9$ \\
\hline & $\mathrm{b}=1.0$ & $b=1.0$ & $\mathrm{~b}=1.0$ & $\mathrm{~b}=1.0$ \\
\hline$p p \rightarrow j j W^{+} W^{-}$ & $\begin{array}{l}95.2 \\
(1820)\end{array}$ & $\begin{array}{l}99.3 \\
(1700)\end{array}$ & $\begin{array}{l}512 \\
(5120)\end{array}$ & $\begin{array}{l}540 \\
(5790)\end{array}$ \\
\hline$p p \rightarrow j j W^{+} W^{-} h$ & $\begin{array}{l}0.011 \\
(0.206)\end{array}$ & $\begin{array}{l}0.0088 \\
(0.172)\end{array}$ & $\begin{array}{l}0.0765 \\
(0.914)\end{array}$ & $\begin{array}{l}0.0626 \\
(0.758)\end{array}$ \\
\hline$p p \rightarrow j j h h h$ & $\begin{array}{l}1.16 \times 10^{-4} \\
\left(3.01 \times 10^{-4}\right)\end{array}$ & $\begin{array}{l}0.0566 \\
(0.0613)\end{array}$ & $\begin{array}{l}0.00115 \\
(0.00165)\end{array}$ & $\begin{array}{l}1.85 \\
(1.46)\end{array}$ \\
\hline
\end{tabular}

Table 2. Cross section (in fb) for $p p \rightarrow j j W^{+} W^{-}, p p \rightarrow j j W^{+} W^{-} h$ and $p p \rightarrow j j h h h$ processes evaluated with Madgraph5. There are two values of the cross sections for each entry, with the number in parenthesis being the cross section without VBF cuts.

\section{Impact of multiparticle production at the LHC and future colliders}

In order to estimate the impact of these enhancements found at the parton level arising from anomalous Higgs couplings, we have performed a full calculation of $p p \rightarrow j j+X$, where $j=u, \bar{u}, d, \bar{d}, s, \bar{s}$ and $X=W^{+} W^{-}, W^{+} W^{-} h, h h h$ at the LHC (for $\sqrt{s}=14$ and $33 \mathrm{TeV}$ ) using Madgraph5 (v1.4.8). We use CTEQ6L1 parton density function for the evaluation of the tree-level cross sections with the QCD scale equal to $M_{Z}$. The selection and acceptance cuts include the requirement of two jets with $P_{T_{j}}>30 \mathrm{GeV}$ with $\left|\eta_{j}\right|<5$ separated with $\Delta R=\sqrt{\Delta \phi_{j j}^{2}+\Delta \eta_{j j}^{2}}>0.4$. Besides the cross sections evaluated for the cuts above we have evaluated another set of the cross sections for an additional cut to select the vector boson fusion process by requiring each jet to be quite energetic with $E_{j}>300 \mathrm{GeV}$ as well as a large rapidity gap between the two jets $\left|\Delta \eta_{j j}\right|>4$ (see e.g. [9] for detailed motivation of this choice). In table 2 we present the results with and without the vector boson fusion cut.

One can notice that when there are gauge bosons in the final state the cross section actually decreases for most cases with $a=1$ versus $a=0.9$ ones. This is because we chose in our example $a<a_{S M}=1$ and since the transverse polarisations dominate the cross section, reducing the coupling $a$ results in a smaller cross section. However, in the case of triple Higgs production, the enhancements are substantial: roughly a factor of 500 for 


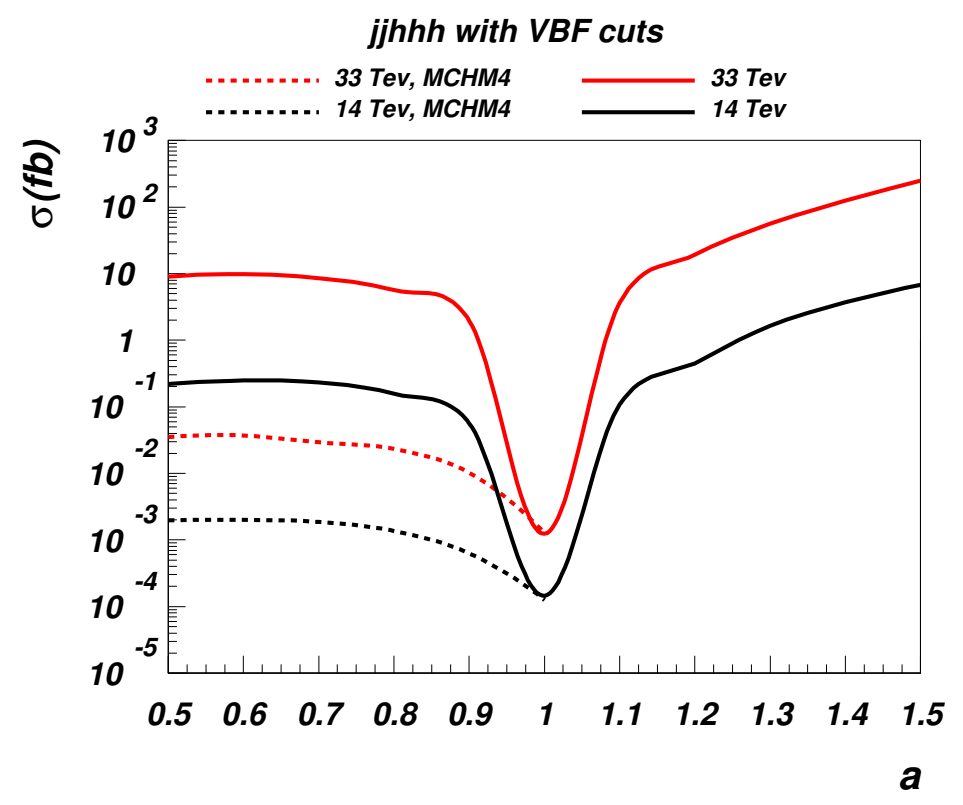

Figure 3. Cross section for triple Higgs production $p p \rightarrow j j h h h$ with VBF cuts as a function of the anomalous coupling $a$ for LHC14 (dark lines) and LHC33 (light lines). Dashed lines are for other parameters fixed to SM values and solid lines are for parameters given by MCHM4 relations.

$\sqrt{s}=14 \mathrm{TeV}$ (LHC14) and 1600 for $\sqrt{s}=33 \mathrm{TeV}$ (LHC33), with VBF cuts. We show in figure 3 the results for the triple Higgs production cross section for both LHC14 and LHC33 for the large range of anomalous coupling $a$. The enhancements with respect to the SM case $a=1$ are large and don't change significantly once $|\Delta a / a|>0.1$.

Though the enhancement can be as large as $10^{5}$ for $a=1.5$, the absolute value of the respective cross sections are quite low (about $10 \mathrm{fb}$ for $\sqrt{s}=14 \mathrm{TeV}$ with VBF cuts) making the study of these processes challenging at the LHC. A dedicated analysis (which are outside the scope of this paper) are necessary to understand the LHC or LHC33 sensitivity to the processes above. However we can already estimate that if such sensitivity is possible it can take place only at high luminocities and/or high energies for quite large values of the $a$ parameter. A detailed analysis of these processes at future $e^{+} e^{-}$colliders is being performed in [19].

\section{Conclusion}

In this article we have studied multiparticle production in models with anomalous Higgs couplings, such as the composite Higgs models. The modified couplings result in a partial unitarization of the scattering amplitudes. We found that, due to the stronger cancellations in the corresponding amplitudes compared to the usual $2 \rightarrow 2$ processes, very large enhancements with respect to the SM, as large as $\mathcal{O}\left(10^{6}\right)$, can arise at the parton level in the cross section of longitudinally polarised gauge bosons, even for small deviations of 
the couplings from their SM values. The cross sections grow faster with energy for larger multiplicities, as expected from naive phase space considerations. We pointed out that some $2 \rightarrow 3$ processes become as important as $2 \rightarrow 2$ processes for relatively low energies, of the order of a $\mathrm{TeV}$, signalling the onset of nonperturbative effects. When accounting for the contributions from the transverse polarisations, the enhancements are somewhat diluted but remain important in some processes, especially triple Higgs production.

However, we showed with a realistic calculation that even with these large enhancements the search for multiparticle processes will remain a challenge for the foreseeable future. On the other hand, multiple gauge and Higgs boson production receives large enhancements in the case of anomalous Higgs boson couplings, and its study could be an important part of future experimental programs aiming at understanding underlying theory beyond the Standard Model.

\section{Acknowledgments}

It is a pleasure to thank C. Grojean, H.-J. He and C. Quigg for helpful discussions. RR and ACAO thank the Theory Division at CERN for the hospitality during the development of this work. RR was supported by a FAPESP grant 2011/10199-3, ACAO by a CAPES fellowship, and MCT by a STFC studentship grant. RR thanks IFT-UNESP for granting a sabbatical leave and the ICTP-SAIFR, funded by a FAPESP grant 11793-4. AB acknowledges partial support from the STFC Consolidated ST/J000396/1 grant as well as from Royal Society grants JP090598 and JP090146.

Open Access. This article is distributed under the terms of the Creative Commons Attribution License which permits any use, distribution and reproduction in any medium, provided the original author(s) and source are credited.

\section{References}

[1] M.S. Chanowitz and M.K. Gaillard, Multiple production of $W$ and $Z$ as a signal of new strong interactions, Phys. Lett. B 142 (1984) 85 [INSPIRE].

[2] V. Rubakov, Nonperturbative aspects of multiparticle production, hep-ph/9511236 [INSPIRE].

[3] D. Morris, R. Peccei and R. Rosenfeld, Multiple $W_{L}$ production from inelastic $W_{L} W_{L}$ scattering at $\sqrt{s} \gg M_{H}$, Phys. Rev. D 47 (1993) 3839 [hep-ph/9211331] [InSPIRE].

[4] U. Aydemir, M.M. Anber and J.F. Donoghue, Self-healing of unitarity in effective field theories and the onset of new physics, Phys. Rev. D 86 (2012) 014025 [arXiv:1203.5153] [INSPIRE].

[5] D.A. Dicus and H.-J. He, Scales of fermion mass generation and electroweak symmetry breaking, Phys. Rev. D 71 (2005) 093009 [hep-ph/0409131] [INSPIRE].

[6] F. Maltoni, J. Niczyporuk and S. Willenbrock, The scale of fermion mass generation, Phys. Rev. D 65 (2002) 033004 [hep-ph/0106281] [INSPIRE].

[7] E. Byckling and K. Kajantie, Particle kinematics, John Wiley \& Sons Ltd., London U.K. (1973). 
[8] G. Giudice, C. Grojean, A. Pomarol and R. Rattazzi, The strongly-interacting light Higgs, JHEP 06 (2007) 045 [hep-ph/0703164] [INSPIRE].

[9] H.-J. He et al., CERN LHC signatures of new gauge bosons in minimal higgsless model, Phys. Rev. D 78 (2008) 031701 [arXiv:0708.2588] [INSPIRE].

[10] R. Contino, C. Grojean, M. Moretti, F. Piccinini and R. Rattazzi, Strong double Higgs production at the LHC, JHEP 05 (2010) 089 [arXiv: 1002.1011] [INSPIRE].

[11] R. Grober and M. Muhlleitner, Composite Higgs boson pair production at the LHC, JHEP 06 (2011) 020 [arXiv: 1012.1562] [INSPIRE].

[12] R. Contino, D. Marzocca, D. Pappadopulo and R. Rattazzi, On the effect of resonances in composite Higgs phenomenology, JHEP 10 (2011) 081 [arXiv:1109.1570] [INSPIRE].

[13] T. Hahn and M. Pérez-Victoria, Automatized one loop calculations in four-dimensions and D-dimensions, Comput. Phys. Commun. 118 (1999) 153 [hep-ph/9807565] [InSPIRE].

[14] J. Alwall, M. Herquet, F. Maltoni, O. Mattelaer and T. Stelzer, MadGraph 5: going beyond, JHEP 06 (2011) 128 [arXiv:1106.0522] [INSPIRE].

[15] N.D. Christensen and C. Duhr, FeynRules - Feynman rules made easy, Comput. Phys. Commun. 180 (2009) 1614 [arXiv:0806.4194] [INSPIRE].

[16] C. Degrande et al., UFO - the Universal FeynRules Output, Comput. Phys. Commun. 183 (2012) 1201 [arXiv:1108.2040] [INSPIRE].

[17] A. Belyaev, N.D. Christensen and A. Pukhov, CalcHEP 3.4 for collider physics within and beyond the Standard Model, Comput. Phys. Commun. 184 (2013) 1729 [arXiv:1207.6082] [INSPIRE].

[18] A. Semenov, LanHEP: a package for the automatic generation of Feynman rules in field theory. Version 3.0, Comput. Phys. Commun. 180 (2009) 431 [arXiv:0805.0555] [InSPIRE].

[19] R. Contino, C. Grojean, D. Pappadopoulo, R. Rattazzi and A. Thamm, Strong Higgs interactions at a linear collider, work in progress. 